\title{
Physical Origin of Anharmonic Dynamics in Proteins: New Insights From Resolution-Dependent Neutron Scattering on Homomeric Polypeptides
}

\author{
Giorgio Schiró, ${ }^{1 *}$ Francesca Natali, ${ }^{2}$ and Antonio Cupane ${ }^{1}$ \\ ${ }^{1}$ Dipartimento di Fisica, Universitá di Palermo, via Archirafi 36, I-90123, Palermo, Italy \\ ${ }^{2}$ CNR-IOM, c/o Institut Laue Langevin, 6 Rue Jules Horowitz, BP 156-38042 Grenoble, France
}

(Received 4 May 2012; published 19 September 2012)

\begin{abstract}
Neutron scattering reveals a complex dynamics in polypeptide chains, with two main onsets of anharmonicity whose physical origin and biological role are still debated. In this study the dynamics of strategically selected homomeric polypeptides is investigated with elastic neutron scattering using different energy resolutions and compared with that of a real protein. Our data spotlight the dependence of anharmonic transition temperatures and fluctuation amplitudes on energy resolution, which we quantitatively explain in terms of a two-site model for the protein-hydration water energy landscape. Experimental data strongly suggest that the protein dynamical transition is not a mere resolution effect but is due to a real physical effect. Activation barriers and free energy values obtained for the protein dynamical transition allow us to make a connection with the two-well interaction potential of supercooledconfined water proposed to explain a low-density $\rightarrow$ high-density liquid-liquid transition.
\end{abstract}

DOI: 10.1103/PhysRevLett.109.128102

PACS numbers: 87.14.ef, 83.85.Hf, 87.15.H-

Neutron scattering is a powerful technique to study protein dynamics and energetics. The structure factor $S(E=0, Q, T)$ of elastic incoherent neutron scattering (EINS) is related to the time-position self correlation function of protein-solvent nuclei that, in turn, is related to the energy landscape of the system whose different tiers can be explored by the temperature dependence of the EINS signal. In particular, EINS on $\mathrm{D}_{2} \mathrm{O}$-hydrated protein powders, which probes the mean square displacements (MSDs) of protein nonexchangeable $\mathrm{H}$ atoms, reveals two deviations from harmonic dynamics, at $\sim 100-150 \mathrm{~K}$ and at $\sim 220 \mathrm{~K}[1,2]$. Molecular origin, physical nature and biological relevance of these "transitions" are still matter of discussion. The first one is attributed mainly to thermally activated motions of $\mathrm{CH}_{3}$ methyl groups [1,3-6] (for this reason hereon called methyl groups activation, MGA). The second one, called "protein dynamical transition" (PDT), has been first interpreted as a glasslike transition [2] directly correlated to the onset of biological activity [7], but this view has been later challenged. Several interpretations of the PDT have been proposed: (i) a change in the protein structural flexibility in response to the glass transition of hydration water [8]; (ii) a result of the protein structural relaxation reaching the limit of the experimental frequency window [9-11]; (iii) the protein response to a fragile-to-strong dynamic crossover in the hydration water at $220 \mathrm{~K}$ where water structure makes a low density liquid $(\mathrm{LDL}) \rightarrow$ high density liquid (HDL) transition [12]; (iv) a change in the thermodynamic resilience of the waterprotein system [13]. These models propose physical pictures partially alternative, demonstrating that the question has not been definitively settled yet: (i) and (ii) ascribe the PDT to a temperature dependent relaxation time crossing the instrumental time-scale; (iii) and (iv) interpret the PDT as a change in thermodynamics and structure of the proteinwater system. All these hypotheses share the relevant role attributed to the hydration water dynamics: in fact, there is clear experimental evidence that the PDT occurs only in the presence of a sufficient amount of hydration water [1,4]. A key issue is whether and how the onset temperature and amplitude of the detected anharmonic MSDs depend on the time scale probed by neutron spectrometers, i.e., on their energy resolution. Indeed, the answer to this question relies on the nature of the underlying energy surface and, as a consequence, gaining information on the resolution dependence helps in describing the shape of the energy landscape. Moreover, it allows us to discriminate among the different hypotheses on the PDT origin listed above: indeed a PDT onset independent of resolution is incompatible with (i) and (ii), while it would support (iii) and (iv). Some EINS studies showing that the PDT onset temperature changes with the energy resolution are controversial in that they concern samples where the solvent signal is non-negligible [14,15] or they are not sufficiently supported by clear experimental data [11]. In fact, experimental problems arise from (i) the lack of a clear definition of MGA and PDT temperatures that makes the identification of "onset temperatures" heavily dependent on the researcher's eye and (ii) the presence in the same sample of both anharmonic activations, often partially overlapping. Moreover, investigations on the resolution dependence of anharmonic MSD amplitudes are lacking.

Here we present new experimental evidence on the resolution dependence of anharmonic onsets; we also propose a physical model that quantitatively and consistently explains the results. Our strategy is to use homomeric polypeptides (HP), i.e., chains of one type of amino acid but a number of residues comparable to that of functional proteins, as model systems to catch the molecular details of 
protein dynamics observed with EINS [3,4]; this overcomes the problem posed by the intrinsic heterogeneity of proteins since it allows us to probe the dynamical behavior of each type of residue separately without neglecting the polymeric nature. We study poly-glycine (poly-G) and poly-alanine (poly-A) in the dry state (where PDT does not occur) and at a hydration level $(h=0.2 \mathrm{~g}$ $\mathrm{D}_{2} \mathrm{O} / \mathrm{g}$ HP) low enough to get a signal attributable to protein nonexchangeable $\mathrm{H}$ atoms with a negligible $\mathrm{D}_{2} \mathrm{O}$ contribution [15], but high enough to allow the PDT. We have recently shown that hydrated poly-G undergoes only the PDT (no $\mathrm{CH}_{3}$ are present) while dry poly-A shows only the MGA $[3,4]$ : in this way we are able to study the resolution dependence of the two transitions, separately, avoiding their superposition. The MSD temperature dependence of these systems is compared to that of a representative globular protein, bovine serum albumin (BSA). The energy resolution dependence is investigated by using three different spectrometers (IN16, IN13 and IN6 at ILL, Grenoble) that allow exploring about two orders of magnitude in energy resolution (FWHM): $0.9 \mu \mathrm{eV}$ (IN16), $8 \mu \mathrm{eV}$ (IN13), and $70 \mu \mathrm{eV}$ (IN6), i.e., the $100 \mathrm{ps}-10 \mathrm{~ns}$ time range. EINS data were analyzed with a standard procedure to obtain the experimental MSDs of nonexchangeable $\mathrm{H}$ atoms: $\mathrm{MSD}=-\partial \ln [S(E=0, Q, T)] / \partial Q^{2}$. Details on experiments and data analysis and the complete set of MSDs are given in the Supplemental Material (SM)[[16]]. To estimate the MGA onset temperatures in dry poly-A, we first subtracted from the MSDs the harmonic contribution extrapolated from the low temperature behavior; then we normalized the obtained $\Delta$ MSDs to their room temperature value. To detect the PDT onset temperature we used an analogous method: the quantities $\Delta \mathrm{MSD}=\mathrm{MSD}_{\text {hydrated }}-$ MSD $_{\text {dry }}$ were first obtained by subtracting from the MSD relative to the hydrated samples [17] those relative to the corresponding dry sample, where PDT does not occur. MSD values relative to dry and hydrated samples are identical up to the PDT onset, see SM. $\Delta$ MSD were then normalized to their room temperature value. It is clear that deviation from zero of the normalized $\triangle$ MSD reveals the MGA onset temperatures in dry poly-A and the PDT onset temperatures in hydrated poly-G; moreover, comparison of data obtained with different spectrometers is no more dependent on the MSD amplitude. The results show that: (i) deviations from the harmonic trend in dry poly-A (Fig. 1(a)) occur at different temperatures: $\sim 110 \mathrm{~K}$ at IN16, $\sim 150 \mathrm{~K}$ at IN13, $\sim 180 \mathrm{~K}$ at IN6; (ii) there is no evidence (Fig. 1(b)), inside the region $215 \pm 10 \mathrm{~K}$, of any PDT onset temperature dependence on the energy resolution. From the results in Fig. 1, we conclude that, within the resolution range explored, the MGA onset temperature clearly depends on energy resolution while the PDT one does not. The same conclusions hold for a real protein, as suggested in Fig. 2 by the temperature dependence of MSDs measured on $\mathrm{D}_{2} \mathrm{O}$-hydrated $(h=0.2)$ BSA at IN16, IN13, and IN6.

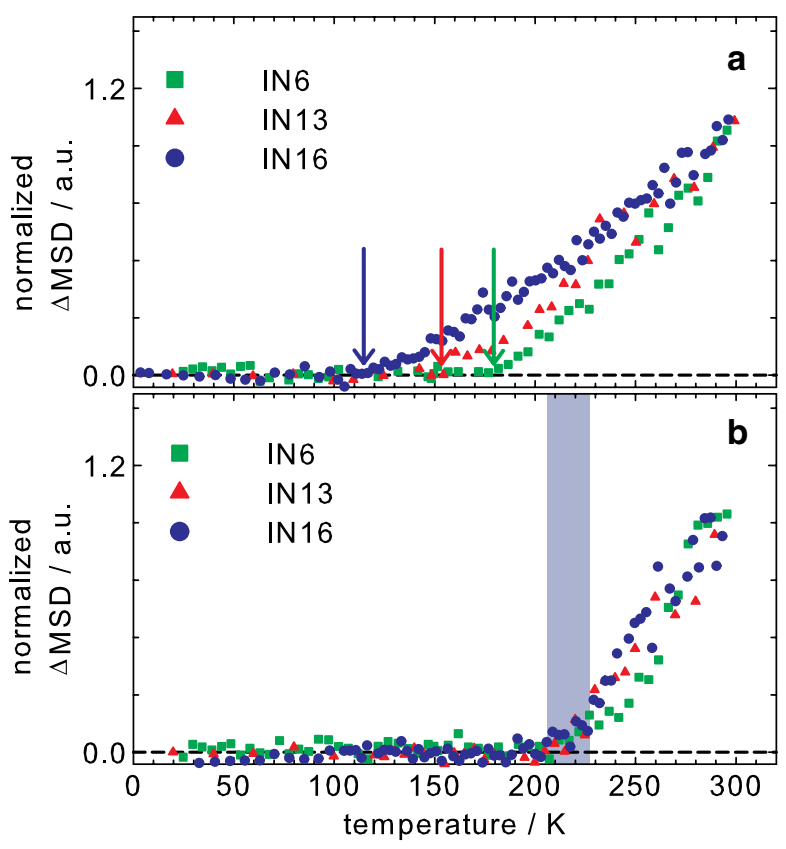

FIG. 1 (color online). Panel (a): normalized $\triangle$ MSD (dryharmonic) in dry poly-A; the arrows indicate MGA temperatures. Panel (b): normalized $\triangle$ MSD (hydrated-dry) in hydrated poly-G; blue (gray) area indicates the PDT onset temperature region.

This experimental evidence excludes the hypothesis of the PDT as a mere resolution effect [8-11].

To analyze quantitatively the experimental data, we introduce an analytical form for the measured observables. First of all the scattering function $S(E, Q, T)$ must be

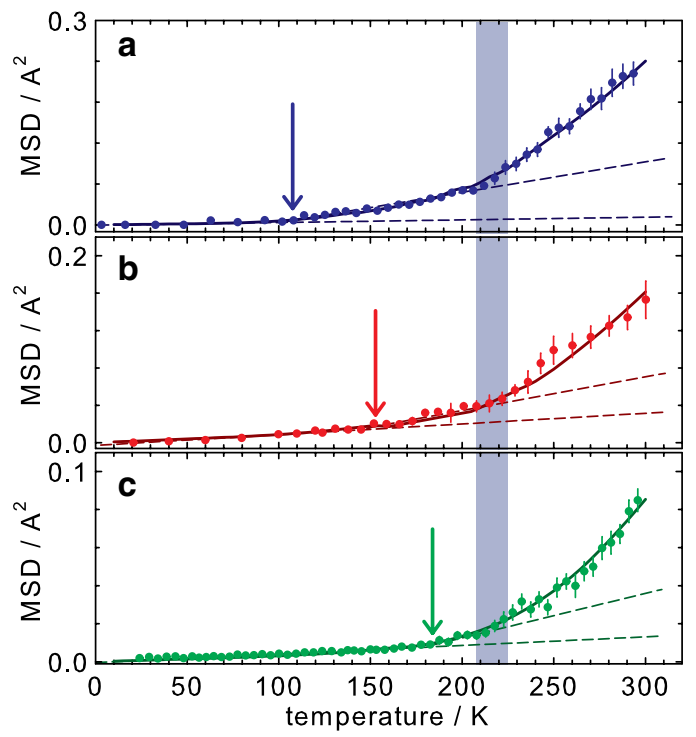

FIG. 2 (color online). Measured (circles) and calculated (lines) MSDs of hydrated BSA (a:IN16, b:IN13, c:IN6). Arrows and blue (gray) areas indicate the MGA and PDT temperatures, respectively. Calculated lines are obtained using the same parameters as in Table I except $\lambda=0.37$ (MGA) and $\lambda=0.21$ (PDT). 
convoluted with the resolution function $R(\mathrm{E} ; \Delta \mathrm{E})$ typical of a spectrometer with $\Delta \mathrm{E}$ resolution:

$$
S_{\text {model }}(E, Q, T)=S(E, Q, T) \otimes R(\mathrm{E} ; \Delta \mathrm{E}),
$$

where $R(\mathrm{E} ; \Delta \mathrm{E})$ is obtained from the totally elastic signal at low temperature and $\otimes$ is the convolution operator in the energy domain. $S(E, Q, T)$ is obtained from an asymmetric two-site energy landscape model of anharmonicity [18], already used by Doster and coworkers to describe anharmonic activations in proteins [2]:

$$
S(E, Q, T)=e^{-Q^{2} u_{v}^{2}}\left(S_{0} \delta(E)+S_{1} \frac{\frac{\tau_{0}}{\pi} \frac{e^{\Delta G^{*} / R T}}{1+e^{\Delta G / R T}}}{1+\frac{\left(E \tau_{0}\right)^{2}}{h^{2}} \frac{e^{2 \Delta G^{*} / R T}}{\left(1+e^{\Delta G / R T}\right)^{2}}}\right),
$$

where

$$
S_{0}=1-2 \frac{e^{-(\Delta G / R T)}}{\left(1+e^{-(\Delta G / R T)}\right)^{2}}\left(1-\frac{\sin (Q d)}{Q d}\right),
$$

and $S_{1}=1-S_{0} \cdot u_{v}^{2}$ is the temperature-dependent mean harmonic vibrational term, $\Delta \mathrm{G}$ is the free energy difference between the two sites at distance $d$, and $\Delta G^{*}$ is the activation barrier for the jumping from the site at lower energy to the other (the motion in the opposite direction, with activation energy $\Delta \mathrm{G}^{*}-\Delta \mathrm{G}$, is also taken into account in Eq. (2); see Fig. 3 for a pictorial representation). From Eq. (1) the $\mathrm{MSD}_{\text {model }}$ can be calculated as: $\mathrm{MSD}_{\text {model }}=-\partial \ln \left[S_{\text {model }}(E=0, Q, T)\right] / \partial Q^{2}$. As evident from Eqs. (1)-(3), the elastic intensity $S_{\text {model }}(E=0, Q, T)$

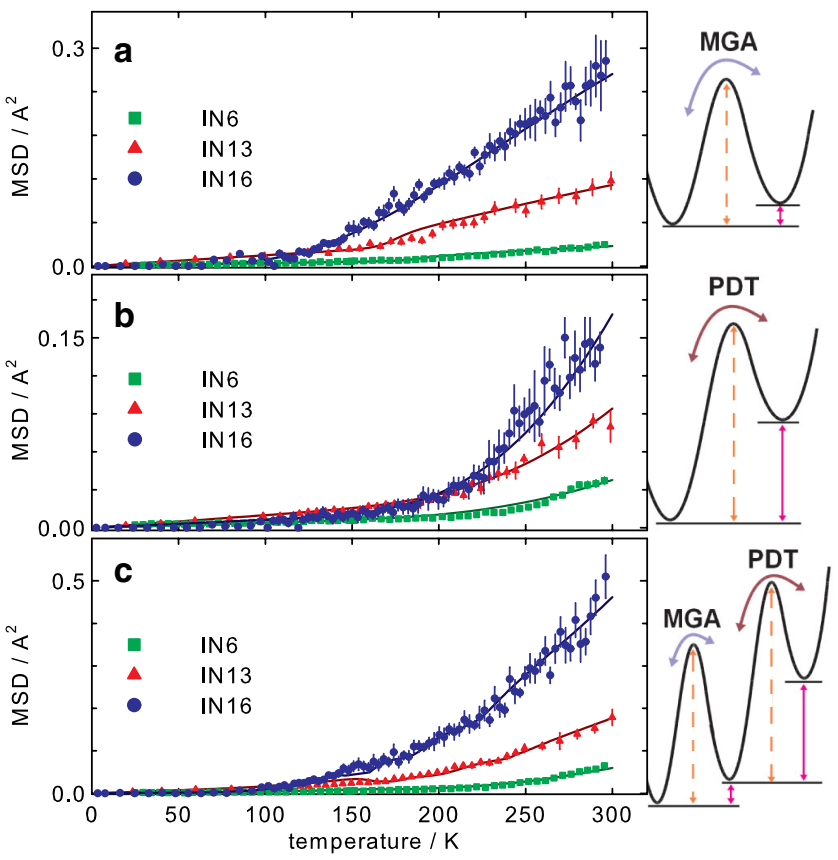

FIG. 3 (color online). Symbols: MSD measured in dry (a) and hydrated (c) poly-A and hydrated poly-G (b); lines: $\mathrm{MSD}_{\text {model }}$ calculated according to the potential pictured on the right (magenta and dashed orange arrows indicate $\Delta \mathrm{G}$ and $\Delta \mathrm{G}^{*}$, respectively). contains also the quasielastic signal falling under the resolution function. As a consequence, a decay with temperature of the elastic intensity can be due to the decrease of $S_{0}$ (which depends only on $\Delta \mathrm{G}$ ) and/or to the broadening of quasielastic peak (which depends also on $\Delta \mathrm{G}^{*}$ ). The first term does not depend upon the energy resolution, while the second does. Thus, one may see that the anharmonic onset temperature described by the two-sites model may change or not with the resolution depending on the $\Delta \mathrm{G}^{*} / \Delta \mathrm{G}$ ratio: (i) for $\Delta \mathrm{G}^{*} / \Delta \mathrm{G} \gg 1$, the appearance of an abrupt activation in the elastic intensity decay is mainly due to the (temperature dependent) broadening of quasielastic width compared to the (temperature independent) resolution width, resulting in a resolution dependent anharmonic onset; (ii) for $\Delta G^{*} / \Delta G \sim 1$, a pronounced decay of $S_{0}$ occurs due to the thermal population of the high energy site, leading to a resolution independent onset. The model is oversimplified in that it assumes homogeneous nonexchangeable $\mathrm{H}$ nuclei, whereas they are chemically and structurally heterogeneous, and it supposes only two energy wells, whereas proteins are characterized by a complex multiminima landscape explored by a random walk within the conformational space [19]. The first issue can be neglected when using homogeneous systems like $\mathrm{HP}$; the second one is here faced introducing an effective distance $d$, related to the length scale explored by $\mathrm{H}$ atoms in the time scale $\tau=h / \Delta \mathrm{E}$ accessed by a spectrometer of resolution $\Delta \mathrm{E}$ via a generalized diffusion-like relation $d=\phi \tau^{\lambda} . \phi$ is related to the diffusion coefficient while $\lambda \leq 0.5$ takes into account a possible subdiffusive behavior, often observed in polymeric systems [20]. The model works if experimental MSDs from different spectrometers can be reproduced by $\mathrm{MSD}_{\text {model }}$ with the same parameters but $\Delta \mathrm{E}$.

Figure 3 reports the MSDs of dry poly-A and of hydrated poly-G (panels $A$ and $B$, respectively), together with the fittings in terms of Eqs. 13, while parameters are reported in Tab. I. A comparison between $S_{\text {model }}(E=0, Q, T)$ calculated by using the parameters reported in Table Iand experimental data in the entire $\mathrm{Q}$ range accessed by IN13 is shown in the SM for poly-A at $T=290 \mathrm{~K}$. We recall that dry poly-A and hydrated poly-G are two systems where only the MGA and the PDT are present, respectively. Figure 3 and Table I show that our model is able to reproduce the entire MSD vs T evolution, from cryogenic

TABLE I. Parameters of the analysis of dry poly-A (MGA) and hydrated poly-G (PDT) data (see text and Fig. 3). Energies are in $\mathrm{kJ} / \mathrm{mol}, \tau_{0}$ in $10^{-20} \mathrm{~s}, \phi$ in $\AA / \mathrm{s}^{2 \lambda}, d$ in $\AA$. $\tau_{0}$ values $\sim 10^{-20} \mathrm{~s}$ are unphysical and reveal a departure from Arrhenius behavior at high temperatures, as discussed in Ref. [21].

\begin{tabular}{lcccccccc}
\hline \hline & $\Delta \mathrm{G}$ & $\tau_{0}$ & $\Delta \mathrm{G}^{*}$ & $\phi$ & $\lambda$ & $d_{\mathrm{IN} 16}$ & $d_{\mathrm{IN} 13}$ & $d_{\mathrm{IN} 6}$ \\
\hline MGA & 5.0 & 2.1 & 44 & $2.0 \times 10^{3}$ & 0.35 & 2.9 & 1.4 & 0.7 \\
PDT & 14 & 2.1 & 21 & $5.0 \times 10^{2}$ & 0.23 & 7.5 & 4.7 & 2.9 \\
\hline \hline
\end{tabular}


to room temperatures, including the resolution dependence of anharmonic onset temperatures and MSD amplitudes. In particular, $\Delta \mathrm{G}^{*} / \Delta \mathrm{G}$ is $\sim 9$ for MGA and $\sim 1.5$ for the PDT, coherently with the difference in the resolution dependence of the onset temperatures. Different values of $\phi$ and $\lambda$ are observed for the different samples: in view of the different structures and hydration conditions, this is an expected result. Moreover, the subdiffusive character $(\lambda<0.5)$ is compatible with that observed in polymers [22]. This result suggests that the observed dependence of PDT-related fluctuation amplitudes on the hydration level $[4,17]$ can be explained with a change in the diffusive properties as a function of water content on the protein surface.

In hydrated HP (like poly-A) and in real proteins (like BSA), both MGA and PDT occur: $\mathrm{CH}_{3}$ rotations and PDTrelated fluctuations are superimposed. Therefore, the scattering function can be obtained from the convolution of the $S_{\text {model }}^{\mathrm{MGA}}(E, Q, T)$ and $S_{\text {model }}^{\mathrm{PDT}}(E, Q, T)$ calculated using the parameters obtained for dry poly-A and hydrated poly-G, respectively. Results are reported in Fig. 3(c) (hydrated poly-A) and Fig. 2 (hydrated BSA). The excellent agreement between data and model further validates the consistency of our analysis and confirms that HP are good model systems for real proteins.

Our results reveal that both anharmonic onsets can be interpreted with similar de-trapping mechanisms (see Fig. 3), but with large differences in their energetics. The MGA is due to a relaxation process between states energetically close (i.e., of a low energy landscape tier, in terms of the H. Frauenfelder's terminology [23]) and without any coupling with the surrounding water matrix; the parameters obtained are in agreement with the current interpretation $[1,3,6]$ of a thermally activated motion between sites with small equilibrium energy differences and with energy barriers and jump distances of the order of $40 \mathrm{~kJ} / \mathrm{mol}$ and $1 \AA$, respectively, as expected for methyl groups rotations. The PDT reflects a transition between states with a large equilibrium free energy difference (i.e., of a higher energy landscape tier) and sustained by the presence of hydration water. Energy barriers are of the order of $20 \mathrm{~kJ} / \mathrm{mol}$ and jump distances of few $\AA$, as expected for backbone and side chain motions. What is the physical mechanism responsible for the water driven effect giving rise to the PDT? A clue is obtained from the fact that the same PDT onset temperature of $\sim 220 \mathrm{~K}$ is observed for chemically and structurally different hydrated peptide systems like native and denaturated proteins, HP (even for poly-G that accounts for the "pure backbone" contribution), amyloid fibrils [24] and amino acid mixtures lacking the polypeptide chain [25]. This implies that the $\Delta \mathrm{G}$ and $\Delta \mathrm{G}^{*}$ values, that set the PDT onset temperature in these systems, are determined by hydration water. The PDT is therefore related to a transition in hydration water, like the LDL $\rightarrow$ HDL transition occurring in supercooled or confined water (like protein hydration water) at $\sim 220 \mathrm{~K}$ (at atmospheric pressure). A model proposed to explain this phenomenon considers an interaction potential characterized by two wells, the outer of which is deeper and narrower [26]. For $T<220 \mathrm{~K}$ water is a one-phase LDL condensed into the narrow well, while for $T>220 \mathrm{~K}$ water can occupy the inner well corresponding to the HDL phase. The activation energy barrier for initiating this structural change [27] corresponds to an H-bond breaking $(\sim 20 \mathrm{KJ} / \mathrm{mol})$; note that it coincides with the $\Delta \mathrm{G}^{*}$ value obtained by our analysis for the PDT. We propose that the protein/hydration water landscape structure revealed by our study and responsible for the PDT is related to the two-wells potential for supercooled water: as proposed by S.-H. Chen and coworkers [28], the LDL $\rightarrow$ HDL transition involves changes in the H-bond network dynamics of hydration water, which is coupled to protein internal motions, thus inducing the PDT-related backbone and side chains fluctuations revealed by EINS.

We are indebted to M. M. Koza and B. Frick for assistance at ILL and to V. Vetri and M. Levantino for discussions. This work was supported by MIUR (Grant No. PRIN2008ZWHZJT).

*giorgio.schiro@unipa.it

[1] J.H. Roh, V. N. Novikov, R. B. Gregory, J. E. Curtis, Z. Chowdhuri, and A. P. Sokolov, Phys. Rev. Lett. 95, 038101 (2005).

[2] W. Doster, S. Cusack, and W. Petry, Nature (London) 337, 754 (1989).

[3] G. Schiró, C. Caronna, F. Natali, and A. Cupane, J. Am. Chem. Soc. 132, 1371 (2010).

[4] G. Schiró, C. Caronna, F. Natali, and A. Cupane, Phys. Chem. Chem. Phys. 12, 10215 (2010).

[5] K. Wood, D. J. Tobias, B. Kessler, F. Gabel, D. Oesterhelt, F. A. A. Mulder, G. Zaccai, and M. Weik, J. Am. Chem. Soc. 132, 4990 (2010).

[6] M. Krishnan, V. Kurkal-Siebert, and J. C. Smith, J. Phys. Chem. B 112, 5522 (2008).

[7] B. F. Rasmussen, A. M. Stock, D. Ringe, and G. A. Petsko, Nature (London) 357, 423 (1992).

[8] W. Doster, J. Non-Cryst. Solids 357, 622 (2011).

[9] S. Khodadadi, S. Pawlus, J.H. Roh, V. G. Sakai, E. Mamontov, and A.P. Sokolov, J. Chem. Phys. 128, 195106 (2008).

[10] G. Chen, P. W. Fenimore, H. Frauenfelder, F. Mezei, J. Swenson, and R. D. Young, Philos. Mag. 88, 3877 (2008).

[11] S. Magazú, F. Migliardo, and A. Benedetto, J. Phys. Chem. B 115, 7736 (2011).

[12] S.-H. Chen, L. Liu, E. Fratini, P. Baglioni, A. Faraone, and E. Mamontov, Proc. Natl. Acad. Sci. U.S.A. 103, 9012 (2006).

[13] G. Zaccai, Science 288, 1604 (2000).

[14] R. M. Daniel, J. L. Finney, V. Réat, R. Dunn, M. Ferrand, and J. C. Smith, Biophys. J. 77, 2184 (1999).

[15] A. Gaspar, S. Busch, M.-S. Appavou, W. Haeussler, R. Georgii, Y. Su, and W. Doster, Biochim. Biophys. Acta 1804, 76 (2010). 
[16] See Supplemental Material at http://link.aps.org/ supplemental/10.1103/PhysRevLett.109.128102 for details on sample preparation, experimental procedures, data reduction and analysis, and supplemental figures.

[17] J. H. Roh, R. M. Briber, A. Damjanovic, D. Thirumalai, S. A. Woodson, and A. P. Sokolov, Biophys. J. 96, 2755 (2009).

[18] A. Stöckli, A. Furrer, Ch. Schönenberger, B. H. Meier, R. R. Ernst, and I. Anderson, Physica (Amsterdam) 136B, 161 (1986).

[19] P. Fenimore, H. Frauenfelder, B. H. McMahon, and F. G. Parak, Proc. Natl. Acad. Sci. U.S.A. 99, 16047 (2002).

[20] H. W. Weber and R. Kimmich, Macromolecules 26, 2597 (1993).

[21] G. Schiró, A. Cupane, S. E. Pagnotta, and F. Bruni, J. NonCryst. Solids 353, 4546 (2007).
[22] A. Arbe, J. Colmenero, M. Monkenbusch, and D. Richter, Phys. Rev. Lett. 81, 590 (1998).

[23] H. Frauenfelder, S. G. Sligar, and P. G. Wolynes, Science 254, 1598 (1991).

[24] G. Schiró, V. Vetri, B. Frick, V. Militello, M. Leone, and A. Cupane, J. Phys. Chem. Lett. 3, 992 (2012).

[25] G. Schiró, C. Caronna, F. Natali, M. M. Koza, and A. Cupane, J. Phys. Chem. Lett. 2, 2275 (2011).

[26] H. Stanley, P. Kumar, S. Han, M. G. Mazza, K. Stokely, S. V. Buldyrev, G. Franzese, F. Mallamace, and L. Xu, J. Phys. Condens. Matter 21, 504105 (2009).

[27] L. Liu, S.-H. Chen, A. Faraone, C. W. Yen, and C. Y. Mou, Phys. Rev. Lett. 95, 117802 (2005).

[28] M. Lagi, X. Chu, C. Kim, F. Mallamace, P. Baglioni, and S.-H. Chen, J. Phys. Chem. B 112, 1571 (2008). 